\title{
Studies on the Chiral Order Parameter in the Schwinger Model
}

\author{
Jen-Fa Min * \\ Department of Physics, National Cheng Kung University, Tainan,Taiwan, 70101, R.O.C \\ Huei-Shih Liao $^{\dagger}$ \\ Department of Physics, National Tsing Hua University, Hsin-Chu, Taiwan, 30043, R.O.C.
}

October 2, 2018

\begin{abstract}
Based on an analytical technique using a unitary transformation and the variational method, we study the chiral order parameter in the Schwinger model in the lattice formalism with KogutSusskind fermions. The fermion condensate $\langle\bar{\Psi} \Psi\rangle$ for any coupling constant and fermion mass are calculated. Chiral symmetry is shown to be broken in the massless limit and good scaling behavior is obtained.
\end{abstract}

*min@ibm65.phys.ncku.edu.tw

†d847301@phys.nthu.edu.tw 


\section{Introduction}

In the field of lattice gauge theory (LGT), there are two major directions of studies. One is to create new techniques such that Monte-Carlo (MC) simulation for systems with fermions so as to get exact results efficiently. The other is to investigate the universality of lattice action and the existence of the continuum limit of LGT.

The Schwinger model[1], which describes $1+1$ dimensional $Q E D$, is superrenomalizable and exactly solvable[1] - [6]. Previous investigations of the model reveal some important properties of $Q C D$ such as quark confinement, chiral symmetry breaking and $U(1)$ problem. LGT with fermions in $1+1$ dimensions is easier to study than in higher dimensions. The study of lattice formalism of the Schwinger model is a good test of the two major directions of LGT. Before we apply the technique to more realistic systems, the studies of the Schwinger model can give us a better understanding of LGT.

The investigations of the Schwinger model have been carried out by MC simulation and by other analytic approach[7] - [14]. Although MC simulation gives several exciting results, analytic methods are still necessary to realize the quatitative picture of its lattice formalism. However, even the finite-lattice technique 15] - 18], one of the most successful analytic methods, is restricted to the strong coupling regime. New analytic approaches still need to be explored.

Recently, a technique with naive fermion formalism[19] is developed. It contains a unitary transformation and the variational method such that the Hamiltonian can be diagonalized and reliable physical informations can be extracted from the Hamiltonian LGT. Our study is concentrated on the Kogut-Susskind formalism of this approach for the Schwinger model. The chiral order parameter $\langle\bar{\Psi} \Psi\rangle$ is calculated and its scaling behavior is investigated.

This paper is organized as follows. In Section 2, we give a brief review of the Kogut-Susskind formalism for the Schwinger model. The unitary transformation and the variational method are introduced in Section 3. Finally, the results and discussion are given in Section 4.

\section{The Schwinger model on a lattice}

We consider the Schwinger model on a lattice and use the Hamiltonian formalism with KogutSusskind fermions. In this formalism, the upper and lower components of the fermion spinor are 
put on different lattice sites. We shall work in the temporal gauge and the Hamiltonian reads

$$
H=-\frac{1}{2 a} \sum_{x}\left[\chi^{+}(x) U(x, x+1) \chi(x+1)+h . c .\right]+m_{0} \sum_{x}(-1)^{x} \chi^{+}(x) \chi(x)+\frac{g^{2}}{2 a} \sum_{x} L^{2}(x),
$$

where $\chi^{+}(x)(\chi(x))$ is a fermionic raising (lowering) operator at site $x, U(x, x+1)$ is the gauge field on the link between site $x$ and $x+1, a$ is the lattice spacing, $m_{0}$ is the bare mass of the fermion and $g$ is the dimensionless bare coupling constant related to the charge by $g=a e . U(x, x+1)$ can be written as

$$
U(x, x+1)=e^{i a e A(x)}=e^{i \theta(x)}=b^{+}(x) .
$$

The dimensionless operator $L(x)$ which generates rotation in $\theta(x)$ is the canonical conjugate to $\theta(x)$ :

$$
\left[L(x), e^{ \pm i \theta(y)}\right]= \pm \delta_{x, y} e^{ \pm i \theta(x)}
$$

Specifying $|n\rangle$ as the eigenstate of $L(x)$, we see that $b^{+}(x)(b(x))$ raises (lowers) the boson number by one:

$$
\begin{aligned}
& b^{+}|n\rangle=|n+1\rangle, \\
& b|n\rangle=|n-1\rangle .
\end{aligned}
$$

We also need the commutation relations between the boson operators and the fermion operators:

$$
\left[\chi^{+}(x), b^{+}(x)\right]=\left[\chi(x), b^{+}(x)\right]=\left[\chi^{+}(x), b(x)\right]=[\chi(x), b(x)]=0 .
$$

The fermion operators $\chi$ and $\chi^{+}$satisfy the usual algebra:

$$
\left\{\chi^{+}(x), \chi(y)\right\}=\delta_{x, y},\left\{\chi^{+}(x), \chi^{+}(y)\right\}=\{\chi(x), \chi(y)\}=0
$$

If we consider the dimensionless parameter $g^{2}$ to be large (strong coupling), the first term in the Hamiltonian can be considered as a perturbed term. The second and the last terms are the unperturbed terms. The ground state $|0\rangle$ of the unperturbed Hamiltonian has the following properties: 


$$
\begin{aligned}
& L^{2}(x)|0\rangle=0, \text { for all } x, \\
& \chi^{+}(x) \chi(x)|0\rangle=\left\{\begin{array}{r}
0, \text { for even } x \\
|0\rangle, \text { for odd } x
\end{array}\right.
\end{aligned}
$$

Because odd lattice sites are occupied by an antiparticle, this state corresponds to a "filled Dirac sea."

The gauge invariant states are specified by fermion occupation numbers and by boson numbers. They are denoted by

$$
|\Psi\rangle=\prod_{x}\left(\chi^{+}(x)\right)^{f(x)}\left(b^{+}(x)\right)^{s(x)}|0\rangle,
$$

where $|0\rangle$ is the "filled Dirac sea," and $f(x), s(x)$ are the numbers of fermions and bosons at location $x$, respectively.

\section{Unitary transformation and the variational method}

We use the unitary transformation:

$$
H^{\prime}=\exp \left(-i \theta S_{f}\right) H \exp \left(i \theta S_{f}\right)
$$

to diagonalize the Hamiltonian. The physical vacuum is assumed to be

$$
|\Omega\rangle=\exp \left(i \theta S_{f}\right)|0\rangle
$$

where

$$
S_{f}=\frac{i}{\sqrt{2}} \sum_{x}(-1)^{x}\left[\chi^{+}(x) U(x, x+1) \chi(x+1)-\chi^{+}(x+1) U^{+}(x, x+1) \chi(x)\right],
$$

and $\theta$ is the variational parameter.

The physical vacuum energy is then given by

$$
E_{\Omega}=\left\langle 0\left|H^{\prime}\right| 0\right\rangle=\left\langle 0\left|\exp \left(-i \theta S_{f}\right) H \exp \left(i \theta S_{f}\right)\right| 0\right\rangle
$$

Defining

$$
H^{\prime}=\exp \left(-i \theta S_{f}\right) H \exp \left(i \theta S_{f}\right)=H_{k}^{\prime}+H_{m}^{\prime}+H_{g}^{\prime}
$$


where

$$
\begin{aligned}
& H_{k}^{\prime}=\exp \left(-i \theta S_{f}\right)\left(-\frac{1}{2 a} \sum_{x}\left[\chi^{+}(x) U(x, x+1) \chi(x+1)+h . c .\right]\right) \exp \left(i \theta S_{f}\right) \\
& H_{m}^{\prime}=\exp \left(-i \theta S_{f}\right)\left(m_{0} \sum_{x}(-1)^{x} \chi^{+}(x) \chi(x)\right) \exp \left(i \theta S_{f}\right) \\
& H_{g}^{\prime}=\exp \left(-i \theta S_{f}\right)\left(\frac{g^{2}}{2 a} \sum_{x} L^{2}(x)\right) \exp \left(i \theta S_{f}\right)
\end{aligned}
$$

and using the Baker-Hausdorff formula

$$
e^{A} F e^{-A}=F+[A, F]+\frac{1}{2 !}[A,[A, F]]+\frac{1}{3 !}[A,[A,[A, F]]]+\ldots \ldots \ldots,
$$

and the commutation relations, we can work out $H_{k}^{\prime}, H_{m}^{\prime}$, and $H_{g}^{\prime}$.

We first consider $H_{m}^{\prime}$. In the expansion of the Baker-Hausdorff formula, we find that, because there is one link in $S_{f}$ and none in $H_{m}^{\prime}$, there appear odd numbers of $U, U^{+}$combinations for the even terms.

$$
\left\langle 0\left|U^{m}\left(U^{+}\right)^{n}\right| 0\right\rangle=0 \text { if } m+n \text { is odd. }
$$

We therefore conclude that

$$
\begin{aligned}
\left\langle 0\left|H_{m}^{\prime}\right| 0\right\rangle & =\sum_{n=0} \frac{1}{(2 n) !}(-\sqrt{2} \theta)^{2 n} m_{0}(-1)^{n}\left(\begin{array}{c}
2 n \\
n
\end{array}\right) \sum_{x}(-1)^{x}\left\langle 0\left|\chi^{+}(x) \chi(x)\right| 0\right\rangle \\
& =-m_{0} \frac{N_{l}}{2} \sum_{n=0} \frac{1}{(2 n) !}(\sqrt{2} \theta)^{2 n}(-1)^{n}\left(\begin{array}{c}
2 n \\
n
\end{array}\right) \\
& =-m_{0} \frac{N_{l}}{2} J_{0}(2 \sqrt{2 \theta}),
\end{aligned}
$$

where $N_{l}$ is the total number of the lattice sites, and $J_{0}(x)$ is the Bessel function of the first kind.

We next consider $H_{k}^{\prime}$. Because there is one link in $S_{f}$ and also one in $H_{k}^{\prime}$, the expansion of the Baker-Hausdorff formula shows that for the odd terms there appear odd numbers of $U, U^{+}$ combinations. These terms cannot exist in $\left\langle 0\left|H_{k}^{\prime}\right| 0\right\rangle$. We finally get

$$
\begin{aligned}
\left\langle 0\left|H_{k}^{\prime}\right| 0\right\rangle & =\sum_{n=0} \frac{1}{(2 n+1) !}(-\sqrt{2} \theta)^{2 n+1} \frac{1}{2 a}(-1)^{n+1}\left(\begin{array}{c}
2 n+2 \\
n+1
\end{array}\right) \sum_{x}(-1)^{x}\left\langle 0\left|\chi^{+}(x) \chi(x)\right| 0\right\rangle \\
& =\frac{1}{2 a}\left(-\frac{N_{l}}{2}\right) \sum_{n=0} \frac{1}{(2 n+1) !}(-\sqrt{2} \theta)^{2 n+1}(-1)^{n+1}\left(\begin{array}{c}
2 n+2 \\
n+1
\end{array}\right) \\
& =\frac{1}{2 a}\left(-\frac{N_{l}}{2}\right) * 2 \sum_{n=0} \frac{1}{n !(n+1) !}(-1)^{n}(\sqrt{2} \theta)^{2 n+1} \\
& =-\frac{1}{a} \frac{N_{l}}{2} J_{1}(2 \sqrt{2 \theta)},
\end{aligned}
$$

with $J_{1}(x)$ being the Bessel function of the first kind.

Finally, we consider the last term $H_{g}^{\prime} . H_{g}^{\prime}$ can be rewritten as 


$$
\begin{aligned}
\left\langle 0\left|H_{g}^{\prime}\right| 0\right\rangle & =\left\langle 0\left|\exp \left(-i \theta S_{f}\right)\left(\frac{g^{2}}{2 a} \sum_{x} L^{2}(x)\right) \exp \left(i \theta S_{f}\right)\right| 0\right\rangle \\
& =\left\langle 0\left|\frac{g^{2}}{2 a} \sum_{x} \exp \left(-i \theta S_{f}\right) L(x) \exp \left(i \theta S_{f}\right) \exp \left(-i \theta S_{f}\right) L(x) \exp \left(i \theta S_{f}\right)\right| 0\right\rangle \\
& =\left\langle 0\left|\frac{g^{2}}{2 a} \sum_{x}[L(x)+B(x)+C(x)][L(x)+B(x)+C(x)]\right| 0\right\rangle,
\end{aligned}
$$

where

$$
\begin{array}{r}
\langle 0|B(x)| 0\rangle=\sum_{n=0} \frac{1}{(2 n+1) !}\left(\frac{\sqrt{2} \theta}{2}\right)^{2 n+1}(-1)^{n+1} \sum_{y}(-1)^{y} \sum_{m=0}^{2 n+1} \sum_{m^{\prime}=m-2 n}^{m}\left(\begin{array}{c}
2 n+1 \\
m
\end{array}\right)\left(\begin{array}{c}
2 n \\
m-m^{\prime}
\end{array}\right) \\
\chi^{+}(y) U^{2 n+1-m}\left(U^{+}\right)^{m} \chi(y+2 n-2 m+1)(-1)^{2 n-2 m+m^{\prime}+2} \delta_{y, x+m^{\prime}} \\
\langle 0|C(x)| 0\rangle=\quad \sum_{n=1} \frac{1}{(2 n) !}\left(\frac{\sqrt{2} \theta}{2}\right)^{2 n}(-1)^{n+1} \sum_{y} \sum_{m=0}^{2 n} \sum_{m^{\prime}=m-2 n+1}^{m}\left(\begin{array}{c}
2 n \\
m
\end{array}\right)\left(\begin{array}{c}
2 n-1 \\
m-m^{\prime}
\end{array}\right) \\
\chi^{+}(y) U^{2 n-m}\left(U^{+}\right)^{m} \chi(y+2 n-2 m)(-1)^{2 n-2 m+m^{\prime}+1} \delta_{y, x+m^{\prime}} .
\end{array}
$$

We now only need to calculate $B^{2}(x)$ and $C^{2}(x)$ in:

$$
\left\langle 0\left|H_{g}^{\prime}\right| 0\right\rangle=\frac{g^{2}}{2 a} \sum_{x}\left[\left\langle 0\left|B^{2}(x)\right| 0\right\rangle+\left\langle 0\left|C^{2}(x)\right| 0\right\rangle\right] .
$$

Here we use the fact that $L(x)|0\rangle=0$ and $\langle 0|B(x) C(x)| 0\rangle=0$. The reason for $\langle 0|B(x) C(x)| 0\rangle=0$ is that $\langle 0|B(x) C(x)| 0\rangle$ involves $\left\langle 0\left|\chi^{+}(y) \chi(y+2 n-2 m+1) \chi^{+}\left(y^{\prime}\right) \chi\left(y^{\prime}+2 n^{\prime}-2 m^{\prime}\right)\right| 0\right\rangle$. The only chance that it will not be zero is that $y^{\prime}=y+2 n-2 m+1$ and $y=y^{\prime}+2 n^{\prime}-2 m^{\prime}$. This implies that $2 n-2 m+1=2 m^{\prime}-2 n^{\prime}$. This is not possible because the left hand side is odd and the right hand side is even. Therefore, we have

$$
\begin{aligned}
\frac{g^{2}}{2 a} \sum_{x}\left\langle 0\left|B^{2}(x)\right| 0\right\rangle= & \frac{g^{2}}{2 a}\left(\frac{N_{l}}{2}\right) \frac{1}{2} \sum_{n=1} \frac{1}{(2 n) !}(\sqrt{2} \theta)^{2 n}(-1)^{n+1}\left(\begin{array}{c}
2 n \\
n
\end{array}\right)\left(\begin{array}{c}
2 n-2 \\
n-1
\end{array}\right), \\
\frac{g^{2}}{2 a} \sum_{x}\left\langle 0\left|C^{2}(x)\right| 0\right\rangle & =\frac{g^{2}}{2 a}\left(\frac{N_{l}}{2}\right)\left[\sum_{n=2} 2^{-3} \frac{1}{(2 n) !}(\sqrt{2} \theta)^{2 n}(-1)^{n}\left(\begin{array}{c}
2 n \\
n
\end{array}\right)\left(\begin{array}{c}
2 n \\
n
\end{array}\right)\right. \\
& -\sum_{n=2} 2^{-2} \frac{1}{(2 n) !}(\sqrt{2} \theta)^{2 n}(-1)^{n}\left(\begin{array}{c}
2 n \\
n
\end{array}\right) \\
& =\frac{g^{2}}{2 a}\left(\frac{N_{l}}{2}\right) \sum_{n=1} \frac{1}{(2 n) !}(\sqrt{2} \theta)^{2 n}(-1)^{n}\left(\begin{array}{c}
2 n \\
n
\end{array}\right)\left[\frac{1}{8}\left(\begin{array}{c}
2 n \\
n
\end{array}\right)-\frac{1}{4}\right] .
\end{aligned}
$$

We wind up with

$$
\begin{aligned}
\left\langle 0\left|H_{g}^{\prime}\right| 0\right\rangle & =\frac{g^{2}}{2 a}\left(\frac{N_{l}}{2}\right) \sum_{n=1} \frac{1}{(2 n) !}(\sqrt{2} \theta)^{2 n}(-1)^{n}\left(\begin{array}{c}
2 n \\
n
\end{array}\right)\left[\frac{1}{8}\left(\begin{array}{c}
2 n \\
n
\end{array}\right)-\frac{1}{4}-\frac{1}{2}\left(\begin{array}{c}
2 n-2 \\
n-1
\end{array}\right)\right] \\
& =\frac{g^{2}}{2 a}\left(\frac{N_{l}}{2}\right)\left\{\frac{1}{8} J_{0}^{2}(2 \sqrt{2} \theta)-\frac{1}{4} J_{0}(2 \sqrt{2} \theta)+\frac{1}{8}-\frac{1}{4} \int_{0} 2 \sqrt{2} \theta \int_{0}^{x} d y d x\left[J_{1}^{2}(y)-J_{0}^{2}(y)\right]\right\} .
\end{aligned}
$$


The dimensionless ground state energy now reads

$$
\begin{aligned}
\epsilon_{\Omega}=\frac{a E_{\Omega}}{N_{l}}= & -\frac{1}{2} m_{0} a J_{0}(2 \sqrt{2 \theta})-\frac{1}{2} J_{1}(2 \sqrt{2 \theta})+\frac{g^{2}}{4}\left\{\frac{1}{8} J_{0}^{2}(2 \sqrt{2} \theta)-\frac{1}{4} J_{0}(2 \sqrt{2} \theta)+\frac{1}{8}\right. \\
& -\frac{1}{4} \int_{0}^{2 \sqrt{2} \theta} \int_{0}^{x} d y d x\left[J_{1}^{2}(y)-J_{0}^{2}(y)\right] .
\end{aligned}
$$

By $\frac{\partial \epsilon_{\Omega}}{\partial \theta}=0$, we can determine the parameter $\theta$ for any coupling constant and bare fermion mass.

\section{Results and Discussion}

Based on the unitary transformation and the variational method we addressed in the last section, we have studied the vacuum structure and chiral symmetry breaking in the Schwinger model with Kogut-Susskind fermions. The reason we prefer to use the Kogut-Susskind formalism is its simplicity.

The fermion condensate $\langle\bar{\Psi} \Psi\rangle$ can be calculated by

$$
\langle\bar{\Psi} \Psi\rangle=\left\langle\Omega\left|\sum_{x}(-1)^{x} \chi^{+}(x) \chi(x)\right| \Omega\right\rangle / N_{l}=-\frac{1}{2} J_{0}(2 \sqrt{2 \theta}) .
$$

Figure 1 plots $\langle\bar{\Psi} \Psi\rangle / g$ as a function of $1 / g^{2}$ in the chiral limit $m_{0}=0$. It shows a very good scaling behavior although the result we calculated, $\langle\bar{\Psi} \Psi\rangle=-0.252 \pm 0.034$, is higher than the exactly calculated result:

$$
\langle\bar{\Psi} \Psi\rangle / e=-\exp (\gamma) / 2 \pi^{3 / 2}=-0.16
$$

Our result would be better if we add the interaction with next to nearest-neighbor term to $S_{f}$. Figure 2 shows $\langle\bar{\Psi} \Psi\rangle$ against the bare fermion mass for $1 / g^{2}=1.0,2.0$, and 3.0, respectively.

For quite a long time, LGT cannot escape the restrict of strong coupling regime. With the application of a unitary transformation and the variational method, the awkward situation of LGT has been overcome. In a future work, we will use the technique to study the chiral symmetry properties and mass spectrum of a two dimensional $S U(2)$ gauge theory coupled to an unflavored Susskind fermion.

This work was supported by the National Science Council of Republic of China under Grant No. NSC 83-0208-M-006-015. We thank Professor Su-Long Nyeo for fruitful discussions. 


\section{References}

[1] Schwinger J., Phys. Rev. 1282425 (1962),

[2] Lowenstein J.H. and Swieca J.A., Ann. Phys. 68172 (1971),

[3] Casher A., Kogut J. and Susskind L., Phys. Rev. D10 732 (1974),

[4] Coleman S., Jackiw R. and Susskind L., Ann. Phys. 93267 (1975),

[5] Fujikawa K., Phys. Rev. Lett. 421195 (1979),

[6] Baaquie B.E., J. Phys.G: Nucl.Phys. 81621 (1982),

[7] Banks T., Kogut J. and Susskind L., Phys. Rev. D13 1043 (1976),

[8] Carroll A., Kogut J., Sinclair D.K. and Susskind L., Phys. Rev. D13 2270 (1976),

[9] Fucito F., Marinari E.,Parisi G. and Rebbi C., Nucl. Phys. B180 369 (1981),

[10] Marinari E., Parisi G. and Rebbi C., Nucl. Phys. B190 734 (1981),

[11] Scalapino D.I. and Sugar R.L., Phys. Rev. Lett. 46519 (1981),

[12] Martin O. and Otto S., Nucl. Phys. B203 297 (1982),

[13] Hamer C.J., Kogut J., Crewther D.P. and Mazzolini M.M., Nucl. Phys. B208 413 (1982),

[14] Ranfit J. and Schiller A., Nucl. Phys. B225 204 (1983),

[15] Crewther D.P. and Hamer C.J., Nucl. Phys. B170 353 (1980),

[16] Hamer C.J. and Barber M.N., J. Phys. A: Math. Gen. 13 L169 (1980),

[17] Hamer C.J. and Barber M.N., J. Phys. A: Math. Gen. 14241 (1981),

[18] Hamer C.J. and Barber M.N., J. Phys. A: Maht. Gen. 142009 (1981),

[19] Luo X.Q. and Chen Q.Z., J. Phys. G: Nucl. Phys. 161181 (1990). 


\section{Figure Captions}

Fig.1 $-\langle\bar{\Psi} \Psi\rangle / g$ was plotted as a function of $1 / g^{2}$ in the chiral limit $m_{0}=0 . g^{2}$ ranges from 0.5 to 10 , where the symbols square and circle represent our results and the exactly calculated value, respectively.

Fig. $2-\langle\bar{\Psi} \Psi\rangle$ was plottted as a function of bare fermion mass $m_{0} a$ for $1 / g^{2}=1.0,2.0$, and 3.0, where the symbols cross, square, and triangle represent $1 / g^{2}=1.0,2.0,3.0$, respectively. 


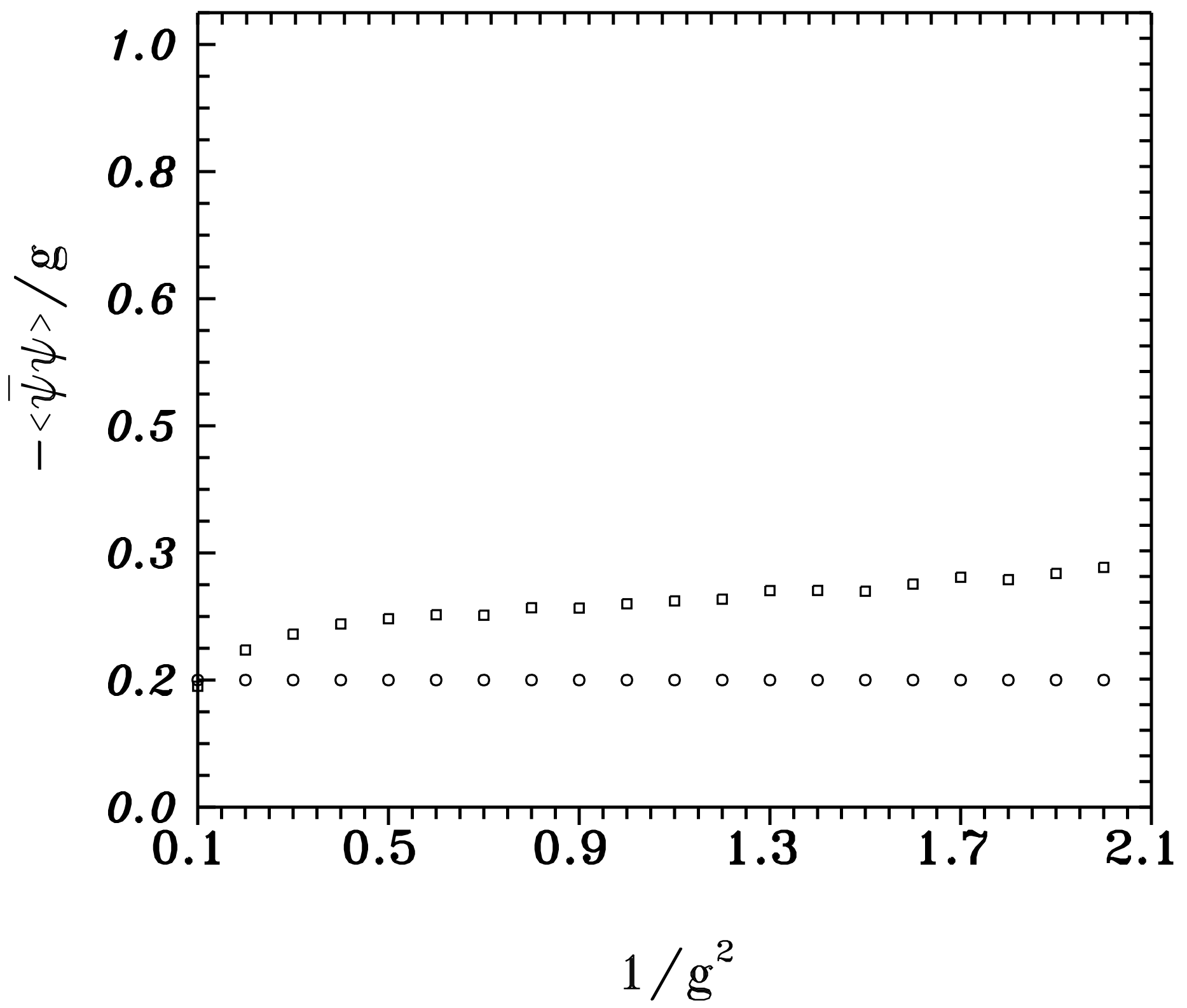

Fig.1 


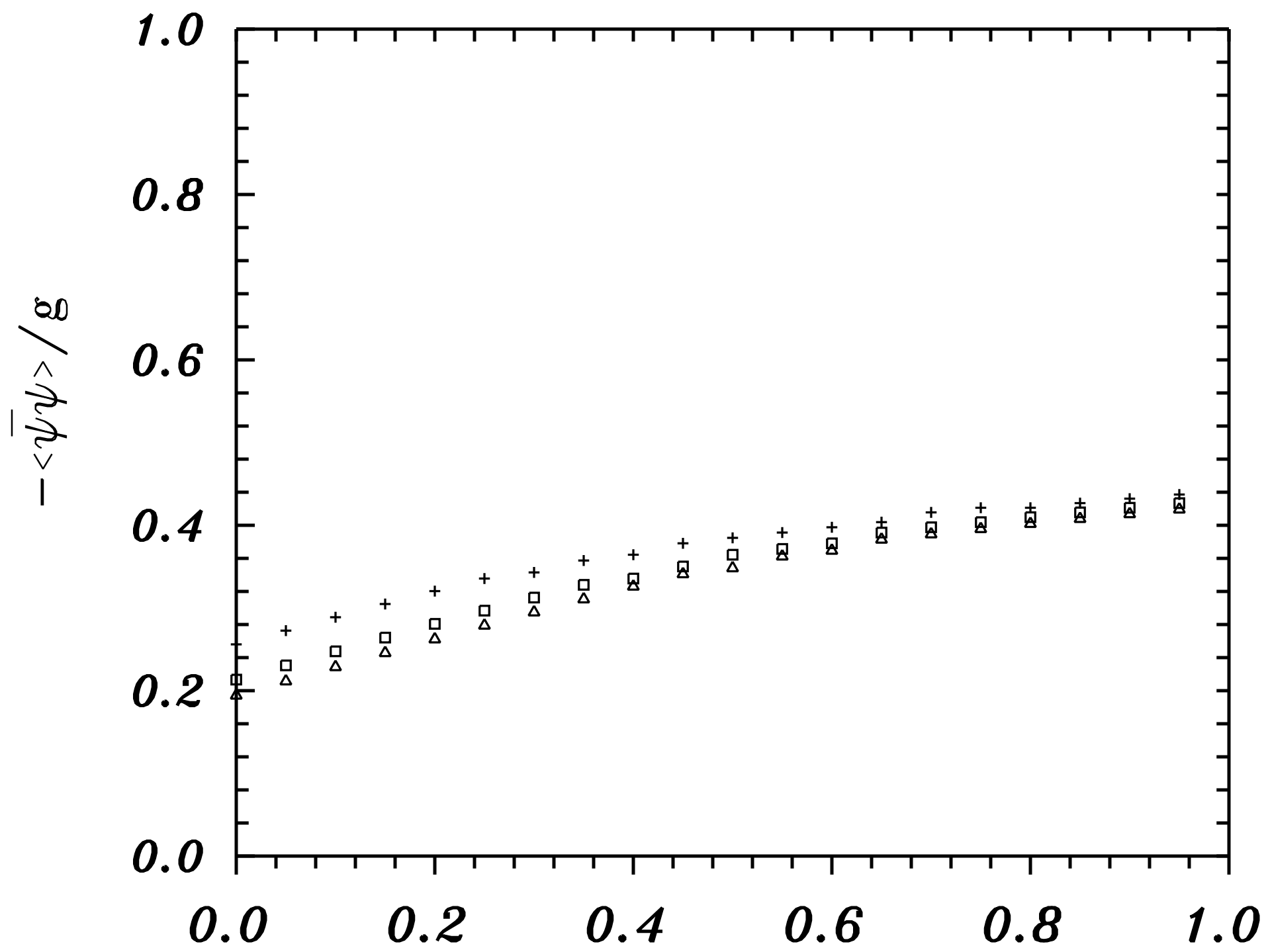

$\mathrm{m}_{0} \mathrm{a}$

Fig.2 